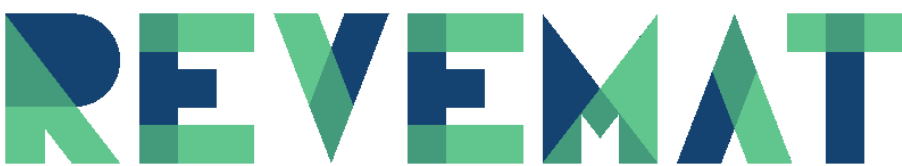

REVISTA ELETRÔNICA DE EDUCAÇÃO MATEMÁTICA

\title{
PROPOSIÇÕES TEÓRICO-SIMBÓLICAS MEDIADAS PELA TEORIA ANTROPOLÓGICA DO DIDÁTICO ${ }^{1}$ Theoretical-symbolic propositions mediated by the anthropological theory of didactics
}

\author{
José Carlos de Souza PEREIRA \\ Secretaria de Educação do Estado do Pará - SEDUC/PA, Belém - PA, Brasil \\ jsouzaper@gmail.com \\ Dhttps://orcid.org/0000-0003-4797-0023 \\ José Messildo Viana NUNES \\ Universidade Federal do Pará (UFPA), Belém - PA, Brasil \\ messildo@ufpa.br \\ Dhttps://orcid.org/0000-0001-9492-4914
} A lista completa com informações dos autores está no final do artigo

\begin{abstract}
RESUMO
O objetivo deste artigo é expor proposições teórico-simbólicas mediadas pela Teoria Antropológica do Didático. As articulações teóricas estabelecidas comtemplam ideias sobre o estudo de obras, sistema didáticos, esquema herbartiano e milieu. A perspectiva dessas articulações estabelece um percurso metodológico para desenvolvimento de pesquisas vinculadas à Didática da Matemática ou Educação Matemática. Nossas proposições teórico-simbólicas são contributos de uma pesquisa de doutoramento. Essa pesquisa envolveu a formação continuada de professores de matemática do Ensino Básico. O dispositivo metodológico dessa formação foi o Percurso de Estudo e Pesquisa. Dispositivo metodológico inerente ao contexto da Teoria Antropológica do Didático, teoria esta, que sustenta nossas proposições teórico-simbólicas.
\end{abstract}

Palavras-chave: Didática da Matemática, Teoria Antropológica do Didático, Proposições Teórico-Simbólicas.

\begin{abstract}
The purpose of this article is to expose theoretical and symbolic propositions mediated by the Anthropological Theory of Didactics. The established theoretical articulations contemplate ideas about the study of works, didactic system, herbartian scheme and milieu. The perspective of these articulations establishes a methodological path for the development of research linked to Didactics of Mathematics or Mathematical Education. Our theoretical-symbolic propositions are contributions of a doctoral research. This research involved the continued training of mathematics teachers for basic education. The methodological device of this training was the Study and Research Course. Methodological device inherent to the context of the Anthropological Theory of Didactics, a theory that supports our theoretical and symbolic propositions.
\end{abstract}

Keywords: Didactics of Mathematics, Anthropological Theory of Didactics, Theoretical-Symbolic Propositions.

${ }^{1}$ Texto adaptado do capítulo teórico da tese de doutoramento do primeiro autor. 


\section{INTRODUÇÃO}

A evolução da Teoria Antropológica do Didático (TAD) alcançou dimensões que ultrapassam o contexto da Didática da Matemática, mas, ainda assim, está centrada na difusão e compreensão dos saberes produzidos pela cultura humana, principalmente, do saber matemático (Chevallard, 1988, 1998, 2002). Nessa evolução, Yves Chevallard e vários pesquisadores, principalmente da França e da Espanha (Catalán, 2003; Delgado, 2006; Matheron, 2000; Briant, 2013; Farras; Bosch \& Gascón, 2013), alargaram os elementos teóricos da TAD a partir de estudos e pesquisas conduzidos em âmbitos institucionais de escolas e universidades.

Esse alargamento dos elementos teóricos da TAD serviu-nos de inspiração para que assumíssemos um percurso metodológico de estudo de diversos artigos de Yves Chevallard, conforme veremos nas seções deste artigo. Esse percurso metodológico é parte da fundamentação teórica da pesquisa de doutoramento do primeiro autor e contém compreensões e proposições teórico-simbólicas vinculadas ao contexto da TAD.

Nossas compreensões e interpretações teóricas estão mediadas por abordagens contidas em artigos de Yves Chevallard nos quais ele específica suas proposições teóricas sobre o que são obras, sistemas didáticos, esquema herbartiano, milieu e várias simbologias inerentes ao corpo teórico da TAD. Nesse sentido, objetivamos, neste artigo, expor proposições teórico-simbólicas mediadas pela Teoria Antropológica do Didático, as quais entendemos ter relevância para pesquisas em Educação Matemática.

\section{O ESTUDO DE OBRAS E SUAS INTERFACES NA TEORIA ANTROPOLÓGICA DO DIDÁTICO}

O estudo das obras é reconhecido por Chevallard (1998) no contexto das organizações didáticas: "As praxeologias didáticas ou organizações didáticas são respostas (no sentido forte) para as questões 'Como estudar a questão $q=\tau_{T}$ ?', ou 'Como estudar a obra $O$ ?' - denotamos aqui as respostas, genericamente, por $\partial q$ e $\partial O$, de tal forma, que se encontrará, por exemplo: $\mathrm{OD}_{\theta}=\partial \mathrm{OM} \mathrm{M}_{\theta}[\ldots]$ ]" (Chevallard, 1998, p. 16, tradução nossa, destaques do autor). Nota-se que a resposta para a tecnologia didática $\left(\mathrm{OD}_{\theta}\right)$ está na tecnologia da Organização Matemática $\left(\partial \mathrm{OM}_{\theta}\right)$. Contudo, a praxeologia didática depende dos tipos de tarefas ou "gestos" didáticos que a torna identificável. O alargamento dessa compreensão está descrito na citação a seguir. 
A questão "Como estudar $\bullet$ ?" depende evidentemente do conteúdo didático $\vee^{2}$. Uma resposta para esta questão, ou seja, uma organização didática $\partial \boldsymbol{\vee}$ dependerá igualmente de $\vee$ : a partir de certo nível de organização de estudo, não se estuda a questão $q$ na perspectiva como se estuda a questão q' da criptografia, por exemplo! Tão pouco se poderá dizer que não haja nada em comum entre uma organização didática $\partial q$ e outra $\partial q^{\prime}$. Com efeito, conforme se tem indicado em uma dada instituição, só alguns tipos de praxeologias didáticas, que satisfazem certas restrições, são ecologicamente viáveis: consequentemente, todas as praxeologias didáticas $\partial \vee$ cumprem estas restrições, seja qual for $\boldsymbol{\vee}$, sem que se possa afirmar a priori que estas restrições não pesam, ecologicamente, sobre os níveis mais específicos da organização de estudo (Chevallard, 1998, p. 16, tradução nossa).

O que se põe na citação remete ao que seja específico, ou não, da obra $\boldsymbol{\bullet}$, isso conforma com as obras de Álgebra Elementar produzidas pela cultura humana, por exemplo, as de Girard (1629, 1884), Viète (1630), Maclaurin (1753), Euler (1795), entre outras. Cada uma dessas obras possui particularidades e semelhanças. Essas obras, ecologicamente, viveram em diferentes épocas, habitaram diferentes instituições e estiveram ajustadas as condições e restrições impostas pela época cultural. Incluam-se nas obras $\boldsymbol{v}$, os Modelos Epistemológicos de Referências (MER) e Modelos Epistemológicos alternativos (MEA). Esses modelos são obras contemporâneas e modificáveis (Gascón, 2011; Farras; Bosch \& Gascón, 2013). O MER possui elementos transpositivos e é "[...] imprescindível para estudar o saber matemático antes que se transforme para ser ensinado [...]" (Farras; Bosch \& Gascón, 2013, p. 5, tradução nossa).

A elaboração de um MER envolve uma dialética de saberes matemáticos, muitos deles contidos em diversas obras • . A obra de Delgado (2006) contém um MER sobre os sistemas de numeração, no qual ele estabelece certa dialética entre a razão de ser dos sistemas de numeração com os sistemas de numeração aditivos, híbridos e posicionais. Outra obra que possui um MER e um MEA, é a de Pereira (2012). Na obra de Pereira (2012) consta a modelização matemática das expressões algébricas polinomiais, vinculadas ao sistema de numeração posicional decimal e de bases quaisquer. Esse MEA elaborado por Pereira revela a existência de um MER para estudar as expressões algébricas polinomiais e suas extensões operatórias, ensinadas no oitavo ano do ensino fundamental (Pereira, 2012; Pereira \& Nunes, 2017). Compreenda-se que esses dois tipos de modelos epistemológicos contemplam fenômenos transpositivos, principalmente, porque está em jogo o saber matemático (Chevallard, 1994; Gascón, 2011; Pereira, 2012; Pereira \& Nunes, 2017). 
No avanço teórico da TAD temos as proposições da "Didactique de l'Enquête Codisciplinaire" [Didática da Investigação Codisciplinar] (Chevallard, 2007, p. 15). Esse tipo de investigação leva em conta as possíveis condições e restrições de uma escala de níveis (co) determinação didática: Civilização $\leftrightarrows$ Sociedade $\leftrightarrows$ Escola $\leftrightarrows$ Pedagogia $\leftrightarrows$ Disciplinas $\leftrightarrows$ Áreas $\leftrightarrows$ Setores $\leftrightarrows$ Temas $\leftrightarrows$ Assuntos (Chevallard, 2007). As problemáticas inclusas na Investigação Codisciplinar estabelecem alguma (s) relação (ões) com os níveis da escala de (co) determinação didática e se apoiam no esquema herbartiano3: $\left(S(X, Y, Q) \rightarrow R_{1}^{\diamond}, R_{2}^{\diamond}, \ldots, R_{n}^{\diamond}, O_{n+1}, \ldots, O_{m}\right) \rightarrow R^{\bullet}$ (Chevallard, 2007). No esquema herbartiano temos o sistema didático $S$, as respostas autenticadas $R_{n}^{\diamond}$ as obras $O_{m}$ e a resposta esperada $R^{v}$. O trabalho para se obter ou elaborar a $R^{v}$ demanda estudo de obras, sejam elas matemáticas ou não matemáticas.

$\mathrm{Na}$ tese de doutoramento do primeiro autor existe um esquema herbartiano adaptado: $\left(\mathrm{S}\left(\mathrm{y}, \mathrm{O}, \mathrm{Q}_{\mathrm{y}}\right) \rightarrow R_{1}^{\diamond}, R_{2}^{\diamond}, \ldots, R_{n}^{\diamond}, O_{n+1}, \ldots, O_{m}\right) \rightarrow R^{\vee}(\mathrm{y}=$ pesquisador; $\mathrm{O}=$ obras matemáticas de diferentes épocas; $Q_{y}=$ questão que motivou o estudo das obras ${ }^{4} ; R_{n}^{\diamond}=$ respostas encontradas nas obras; $O_{n}=$ obras examinadas de diferentes épocas e $R^{v}=$ resposta confirmada por y). Esse esquema herbartiano adaptado resume uma etapa metodológica desenvolvida na execução da pesquisa, ou seja, um roteiro menor para filtrar as ideias essenciais das obras consultadas.

Na constituição teórica da TAD há a "noção de oferta praxeológica5" (Chevallard, 2008, p. 7) que estabelece certo "diálogo" com as tarefas de um projeto de atividade, nas quais o problema do equipamento praxeológico útil e, efetivamente, acessível e está "[...] sob condições apropriadas, compatíveis com as restrições existentes" (Chevallard, 2008, p. 7, tradução nossa), por exemplo, a formação continuada de professores de matemática do Ensino Básico mediada por uma obra (Pereira, 2017). Nossa compreensão é que essa formação deve imprimir alterações ou recombinações praxeológicas no equipamento

\footnotetext{
3 Johann Friedrich Herbart nasceu em 4 de maio de 1776 na cidade de Oldenburg, situada ao norte da Alemanha, e morreu em 11 de agosto de 1841 na cidade universitária de Göttingen. Entre 1794 e 1797, foi aluno do filósofo Johann Gottlieb Fichte (1762-1814) na Universidade de lena. No entanto, o jovem Herbart rapidamente tomará distância da "teoria da ciência" e da filosofia prática de seu mestre. No terreno fértil das contradições do pensamento idealista, fará germinar sua própria filosofia realista. Herbart, no entanto, permanecerá em sua vida inteira fiel ao rigor intelectual de seu mestre Fichte, tentando, a exemplo dele, apresentar os elementos mais importantes de sua reflexão sob a forma de "deduções" (Hilgenheger, 2010, p. 12).

4 "Quais características das organizações praxeológicas da álgebra elementar escolar identificamos em obras de diferentes épocas?" (Pereira, 2017, p. 52).

5 1. La notion d'offre praxeologique
} 
praxeológico desses professores (Chevallard, 2009a; Pereira, 2017), partícipes dessa formação. Deve-se entender que essa oferta ou proposição praxeológica leva em conta as condições e restrições da problemática funcional, anunciada por Chevallard (2008): "Dadas as restrições impostas sobre um conjunto de condições existentes ou a ser criado, qual equipamento praxeológico é possível que uma instituição ou uma determinada pessoa utilize para agir em uma determinada área de atividade?" (Ibidem, 2008, p. 7, tradução nossa). A existência das restrições operando sobre o conjunto de condições reflete na distinção entre estas. As restrições são condições consideradas, possivelmente, não modificáveis, enquanto as condições menores são vistas como modificáveis ou criadas sob as restrições existentes (Chevallard, 2008). A amplitude das restrições e condições alcança as ofertas praxeológicas das obras e a metodologia do Parcours d'Étude et de Recherche (PER) [Percurso de Estudo e Pesquisa (PEP)] (Chevallard, 2011a; Matos, 2017; Pereira, 2017).

As condições e restrições estão no estudo das obras de Álgebra Elementar, ou seja, temos a problemática denominada "de base", assim descrita por Chevallard e Bosch (2012):

Admitida por numerosas pesquisas em didática da álgebra, a problemática de base pode ser anunciada assim: "Dadas às restrições $K$ (por exemplo, as do atual ensino secundário) que são impostas sobre uma instância $U$ (por exemplo, um grupo de alunos e seu professor), sob quais conjuntos de condições $C$ esta instância poderá encontrar a entidade praxeológica $\wp$ (por exemplo, tal organização vista como relevante para a álgebra ensinada)?" No que segue, nós nos referiremos, não à problemática de base, mas a sua problemática dual, a problemática possibilística, que se enuncia nos seguintes termos: "Dado um conjunto de condições $C$ e um conjunto de restrições $K$, aos quais tal instância $U$ é submetida, quais entidades praxeológicas $\wp$ são possíveis que tal instância $U$ conheçam?" Não é assim uma pergunta que se deseja fazer como os alunos podem conhecer (e de que forma seu professor pode fazê-los conhecer) tal ou qualquer entidade "algébrica", mas para identificar os tipos de entidades "algébricas" que é possível encontrarmos hoje na escola, sob as restrições e condições existentes. Tal perspectiva nos levará, naturalmente, abordar a problemática contrária, dita impossibilística - quais são as entidades praxeológicas $\wp$ de um determinado tipo, sob restrições $K$ e condições $C$, a instância U não encontraram? (Chevallard \& Bosch, 2012, p. 21, tradução nossa).

A obra de Pereira (2017) mostra que a problemática "de base", concernente à Álgebra Elementar Escolar, possui a instância $U$ (professores de matemática do Ensino Básico), submetida ao conjunto de condições $C$ (local, materiais e orientações adequados ao processo de formação continuada). A instância $U$ manifesta as restrições $K$ (de sua formação inicial e outras, assim como, de suas práticas docentes) internalizadas em seu equipamento praxeológico, em relação à entidade praxeológica $\wp$ (MEA contido em 
Pereira (2012)). Nessa mesma obra de Pereira, a problemática possibilística ressoa sobre o estudo que a instância $U$ realizou da Organização Matemática e Didática do MEA contido em Pereira (2012), nela há as condições $C$ (etapas detalhadas da modelização numérico-algébricas a partir das noções de valor posicional e representação na escrita polinomial de potência de base dez) e as restrições $K$ (modelização restritiva ao sistema de numeração posicional decimal e sua extensão para as expressões algébricas de uma única variável); nesse processo de estudo a instância $U$ encontrou e conhece as instâncias praxeológicas $\wp$ inerentes às práticas aritméticas (operações aritméticas fundamentais e seus algoritmos) e práticas algébricas (cálculo algébrico com operações polinomiais).

A problemática "de base" e possibilística é anunciada, de forma abrangente, por Chevallard em 2009, durante as sessões do "Seminário da Teoria Antropológica do Didático -TAD \& Engenharia Didática do Desenvolvimento - EDD6", cujos textos estão publicados no JOURNAL DU SEMINAIRE TAD/IDD 2008/20097. Nesse seminário Chevallard aborda o contexto das didáticas "específicas", a exemplo da didática da matemática (Chevallard, 2009b). A especificidade da didática da matemática "é assim esta parte da ciência didática que estuda a difusão - e a não difusão - das praxeologias matemáticas. Poder-se-á assim estudar as praxeologias que tal instituição / contribui para difundir - sua oferta praxeológica [...]" (Chevallard, 2009b, p. 2, tradução nossa). O descrito na citação pode configurar-se na metodologia do PER $^{8}$ (metodologia que mediou a formação continuada de professores de matemática do ensino básico) que consta na obra de Pereira (2017) e serviu para difundir a oferta praxeológica das obras de Carvalho e Pereira (2009) e de Pereira (2012). Nesse processo de difusão praxeológica ocorreu que a instância $V$ (diretor de estudo) promoveu um "gesto didático", ou seja, a instância $V$ teve que estimular com questionamentos a instância $U$ (professores de matemática do ensino básico) para que esta iniciasse um processo de estudo de algumas obras (artigos sobre a TAD, monografia de Carvalho e Pereira (2009), dissertação de Pereira (2012), entre outras) (Chevallard, 2009b). Chevallard denota essa situação por \#(U; V; $\boldsymbol{\vee})$, ou seja, a problemática "de base" e "possibilística" do processo de formação flui se o diretor de estudo (instância $V$ ) conseguir que os professores de matemática em formação continuada (instância $U$ ) estudem as obras indicadas e externalizem sob que condições $C$

\footnotetext{
${ }^{6}$ Séminaire Théorie Anthropologique du Didactique-TAD \&Ingénierie Didactique du Développement-IDD

7 Disponível em: http://yves.chevallard.free.fr/spip/spip/article.php3?id_article=140 Acesso em: 27 mar. 2016.

8 Parcours d'Étude et de Recherche (PER) (Chevallard, 2009c, 2009d)
} 
e restrições $K$ eles compreendem os conteúdos dessas obras, ou seja, quais praxeologias $\wp$ esses professores objetivam em seus equipamentos praxeológicos.

Em dialética com a notação \#(U; $V ; \boldsymbol{\vee})$ temos a noção de sistema didático, conforme está em Chevallard (2009b): “ [...] a noção de sistema didático, que denoto por $S(X ; Y ; \vee)$, onde $\vee$ é uma obra que $X$ estuda com ajuda de $Y$ " (Ibidem, p. 4, tradução nossa). Ainda segundo Chevallard (2009b): "a noção de sistema didático é integrante da noção de escola: uma escola é uma instituição que oferece de maneira declarada um habitat para certos tipos de sistemas didáticos" (Ibidem, p. 4, tradução nossa). Por exemplo, o habitat de um sistema didático pode ser o curso de doutorado de uma Instituição de Ensino Superior. Para complementar a compreensão sobre sistema didático, Chevallard (2009b) acrescenta:

Assim, no que diz respeito à formação do sistema didático $S(X ; Y ; \vee)$. Deve-se imaginar que alguma instância $V$ (tem-se, em alguns casos, $V=Y$ ou mesmo $V=X$ ) pode modificar de alguma forma a relação de $U=X$ para $\bullet$, isso provocará a constituição de um sistema didático, permitindo que $X$ estude $\bullet$ com ajuda de $Y$.

O funcionamento - o "trabalho" - de um sistema didático supõe um tempo e um lugar próprio, excluídas as atividades "regulares" (não consagradas ao estudo) da vida social dos $x \in X$, pelo qual a atividade no interior de $S(X ; Y ; \boldsymbol{\vee})$ aparece como um lazer de estudos, o que é a mesma definição grega da noção clássica de skholê (palavra de onde derivam as palavras "europeias" schola, école, school, escuela, schule, skole etc.) (Ibidem, p. 4, tradução nossa).

Chevallard (2011b) acrescenta que para a problemática da didática: "Estudar o equipamento praxeológico de uma determinada instância não é uma problemática principal, mais uma problemática derivada (Ibidem, p. 8, tradução nossa). Entretanto, ele esclarece que: "o objetivo principal da didática é o estudo das condições e restrições sob as quais um tipo de equipamento praxeológico de determinada instância se modificou, ou está se modificando ou poderá ser alterado com a integração de tal ou qualquer entidade praxeológica" (Chevallard, 2011b, p. 8, tradução nossa). A partir dessa descrição de Chevallard (2011b), compreendemos que o estudo de obras intercala as duas problemáticas da didática (principal e derivada). Nesse sentido, a formação continuada de professores de matemática do ensino básico, mediada por um MEA possui essa problemática (Pereira, 2017). Isso porque a análise do equipamento praxeológico objetivado desses professores, revela as condições e restrições existentes para promover as possíveis alterações ou recombinações praxeológicas nos equipamentos praxeológicos desses professores (Pereira, 2017). Existem ainda as condições e restrições do dispositivo metodológico escolhido para conduzir o processo de formação 
continuada, ou seja, o PER. O PER tem a potencialidade de gerar material analisável pela ferramenta indicada por Chevallard (2011b):

A ferramenta de tais estudos é a análise didática: análise do didático que conduz, ou impulsiona, ou poderá suscitar a visada mudança praxeológica. A análise didática supõe a análise de condições e restrições de todos os níveis, em particular, a análise praxeológica de obras, ou seja, de entidade praxeológica da qual se estuda, também, a difusão passada, presente e futura. Inversamente, a análise praxeológica é chamada análise didática. Esclarecer o que tal instância "sabe" - ou seja, o que contém seu equipamento praxeológico - para tal ou qualquer propósito supõe em muitos casos uma análise genética, revelando como ele aprendeu, e de que, portanto, supõe-se uma análise didática.

O precedente leva a uma conclusão inesperada: em uma perspectiva de crítica cidadã, qualquer que seja o propósito de se "aprender" tal entidade praxeológica $\checkmark$ (na família, na escola, na mídia, etc.), deve-se perguntar o que é $\boldsymbol{\vee}$, ou seja, realizar uma análise praxeológica de $\mathbf{v}$; ou para elucidar o que a instância docente assim lhe oferece para o saber $\boldsymbol{v}$, frequentemente, convém determinar como essa própria instância sabe ou aprendeu essa entidade praxeológica. A análise didática, não menos que a análise praxeológica, é assim uma ferramenta "cidadã", na qual a escola deve formar as próximas gerações. (Chevallard, 2011b, p. 8-9).

O último parágrafo da citação precedente, Chevallard (2011b) encaminha uma ideia que merece estudo e pesquisa, é uma proposição que alia análise didática e análise praxeológica, isso na perspectiva de que a escola forme os sujeitos das gerações futuras mais hábeis quanto cidadãos analíticos.

Nesta seção mostramos algumas interfaces do estudo de obras no contexto TAD. $\mathrm{Na}$ seção seguinte, expomos algumas proposições teórico-simbólicas que elaboramos durante o desenvolvimento da pesquisa de doutorado do primeiro autor.

\section{PROPOSIÇÕES TEÓRICO-SIMBÓLICAS MEDIADAS PELA TAD}

Nesta seção, o estudo e as simbologias de obras no contexto da TAD, serão mediados por compreensões e interpretações que idealizamos como parte complementar do quadro teórico da pesquisa de doutoramento do primeiro autor.

Nossas interpretações simbólicas de obras específicas mediaram o processo de formação continuada e as sessões da metodologia do PER: $\boldsymbol{\vee}_{\mathfrak{M}}=$ Monografia de Carvalho e Pereira (2009), $\boldsymbol{\vee}_{\mathfrak{D}}=$ Dissertação de Pereira (2012), $\boldsymbol{\vee}_{\mathfrak{E}}=$ Modelo epistemológico Alternativo de Pereira (2012). Além disso, usamos a simbologia $\vee_{\tau}$ para designar a obra final, a tese doutoral. Essas notações simbólicas nos permitiram adaptar os seguintes sistemas didáticos: $S\left(U ; V ; \vee_{T}\right)=\left(\right.$ doutorando; orientador; tese) e $S\left(U ; V ;\left[\vee_{\mathfrak{M}}, \vee_{\mathfrak{D}}, \vee_{\mathfrak{E}}\right]\right)=$ (professores de matemática participantes do PER; diretores de estudo; obras principais). 
As simbologias dos dois sistemas didáticos nos auxiliaram nas análises do processo de formação continuada e na elaboração da resposta para a questão principal da pesquisa (denotada pela simbologia $Q$ ).

Essas proposições simbólicas estão inspiradas em Chevallard (2011c, p. 1) que estabelece a conexão do sistema didático $S(X ; Y ; \vee)$ com a proposição "Étudier/chercher $(X)$, diriger l'étude/la recherche $(Y)$ " [Estudar/pesquisar $(X)$, dirigir o estudo/ a pesquisa $(Y)$ ]. O desdobramento dessa conexão está transcrito na citação a seguir.

Um pesquisador $x$ estuda uma questão $Q$ ou, geralmente, uma obra $O$ (recordemos que uma questão é uma obra). Designamos pelo símbolo vesta questão $Q$ ou esta obra; nosso pesquisador $x$ forma um sistema didático que, em alguns casos, escreve-se $S(x ; \emptyset ; \vee)$, outros $S(X ; \emptyset ; \bullet)$ com $x \in X$ : aqui, em princípio, os $y \in Y$ não participam de forma central da pesquisa, mas a acompanha. No caso da direção por y do trabalho de tese do "jovem pesquisador" $x$, teremos, geralmente, $S(x ; y ; \boldsymbol{\vee})$; mas poderemos ter também $S(\{x, y\} ; y ; \boldsymbol{\vee}): x$ e y pesquisam em conjunto, o outro $y$ assume a direção da pesquisa. (Chevallard, 2011c, p. 2, tradução nossa).

Com base na citação acima ajustamos nossa modelização ${ }^{9}$ do sistema didático $S$ para conformar o símbolo $\bullet$ a questão $Q$ norteadora da pesquisa. Nessa modelização de $S$ temos a simbologia $\vee_{\mathrm{\tau}} \rightleftarrows Q$, significando que a obra $\boldsymbol{\vee}_{\mathrm{\tau}}$ leva a responder $Q$ e $Q$ confirma a tese da pesquisa. Assim, surge a relação $R(x, Q)$ e o sistema didático $S(x ; y$; $v_{\tau} \rightleftarrows Q$ ), no qual $x=$ doutorando e $y=$ orientador.

Os sistemas didáticos modelados pela notação $S(X ; Y ; Q)$ deve conduzir a construção de uma resposta $R^{\vee}$ (elaborada ou procurada) para Q (Chevallard, 2011d); uma das proposições de Chevallard para construção dessa resposta interliga-se ao esquema herbartiano compactado: $[S(X ; Y ; Q) \rightarrow M] \leadsto R^{\vee}$ (Chevallard, 2011d). Nesse esquema compactado o sistema didático $S(X ; Y ; Q)$ depende do milieu $u^{10}$ $M=\left\{R_{1}^{\diamond}, R_{2}^{\diamond}, \ldots, R_{n}^{\diamond}, O_{n+1}, \ldots, O_{m}\right\}$. O milieu $M$ contém as respostas prontas $R_{n}^{\diamond}$ e as obras $O_{k}$ (simbologias inerentes a TAD). Muitas dessas respostas prontas estão contidas nas obras $\mathrm{O}_{k}$ (Chevallard, 2011d). Assim, no desenvolvimento de uma pesquisa, Chevallard (2011d) indica cinco gestos básicos relacionados às respostas $R^{\diamond}$ e $R^{\vee}$ :

1) Observar as respostas $R^{\diamond}$ contidas na cultura e práticas sociais.

2) Analisar, o duplo plano experimental e teórico, nestas respostas $R^{\diamond}$.

3) Avaliar estas mesmas respostas $R^{\diamond}$.

\footnotetext{
9 Entender que a modelização é um processo que pode englobar a modelagem matemática, mas aqui, refere-se à proposição de um modelo ou esquema.

10 Palavra que significa "meio" em língua portuguesa, mas no contexto da TAD, o significado é amplo, por isso a usaremos no idioma francês.
} 
4) Desenvolver uma resposta própria $R^{*}$.

5) Difundir e defender a resposta $R^{*}$ assim produzida (Chevallard, 2011d, p. 9, tradução nossa).

Os "cinco gestos básicos" norteiam o mecanismo de procura de $R^{\diamond}$, conexo ao "trabalho de investigação sobre as eventuais respostas $R^{\natural}$, que não está dissociado do trabalho de elaboração da resposta $R^{*}$ procurada" (Chevallard, 2011d, p. 9). O que abstraímos da citação se inter-relaciona com o esquema herbartiano ampliado: $[S(X ; Y$; Q) $\left.\rightarrow\left\{R_{1}^{\diamond}, R_{2}^{\diamond}, \ldots, R_{n}^{\diamond}, O_{n+1}, \ldots, O_{m}\right\}\right] \rightarrow R^{\boldsymbol{v}}$. Evidencia-se nessa ampliação do esquema herbartiano, que os conjuntos $X$ e $Y$ devem investigar a existência das respostas $R^{\natural}$, presentes na cultura e práticas sociais, transcritas ou não nas obras $O_{k}$. De certa maneira, fizemos isso no estudo das obras de Álgebra Elementar (mencionadas na segunda seção do artigo), para o qual modelamos o sistema didático $S\left(y, O, Q_{y}\right)$ e examinamos algumas obras de diferentes épocas, de tal modo que adaptamos o esquema herbartiano: $[S(y, 0$, $\left.\left.\mathrm{Q}_{y}\right) \rightarrow M\right] \rightarrow R_{y}^{\diamond}$. A resposta $R_{y}^{\diamond}$ significa uma resposta particular autenticada por $y$ para a questão $Q_{y}$. A seta $\rightarrow$ significa que o milieu $M$ está constituído de respostas $R_{i}^{\natural}$ e obras $O_{k}$, enquanto a seta $\rightarrow$ indica a elaboração de respostas $R_{i}^{\ominus}, R^{\diamond}$ ou $R^{\vee}$.

Chevallard (2012-2013), ao expor sobre Enquête codisciplinaire \& EDD ${ }^{11}$ [Investigação codisciplinar \& EDD], torna mais explícito o significado dos elementos do esquema herbartiano expandido: "Recordaremos primeiro o esquema geral da investigação sobre uma questão $Q$ - ou o estudo de uma questão $Q$-, dito esquema herbartiano expandido: $[S(X ; Y ; Q) \rightarrow M] \rightarrow R^{\mathbf{v}}$, com $M=\left\{R_{1}^{\diamond}, R_{2}^{\diamond}, \ldots, R_{n}^{\diamond}, O_{n+1}, \ldots, O_{m}\right\}$, (Chevallard, 2012-2013, p. 1, tradução nossa). O esquema herbartiano expandido compreende que:

$M$ design um milieu didático ou milieu para o estudo, ou seja, o conjunto de ferramentas e recursos reunidos pelo sistema didático $S(X ; Y ; Q)$ em vista de construir a resposta $R^{*}$ procurada. O milieu é constituído de obras - ou seja, de criações humanas intencionais, tendo uma finalidade - distinguindo-se do que precede somente duas grandes categorias: de um lado, as respostas $R_{1}^{\diamond}, R_{2}^{\diamond}, \ldots, R_{n}^{\diamond}$ para a questão $Q$ existente nas instituições da sociedade; de outro lado, as outras obras, $O_{n+1}, \ldots, O_{m}$. A natureza dessas obras será especificada de acordo com a necessidade; em regra geral, ver-se-á que elas virão de diversos campos de conhecimento: portanto, fala-se de investigação codisciplinar a propósito das investigações que teremos para conduzir. Refinamos um pouco a modelização do milieu $M$, fazendo aparecer, explicitamente, entre as "outras obras", um tipo de obra essencial: as questões que são geradas pelo estudo da questão $Q$ e, também, pelo

\footnotetext{
11 Éducation au développement durable (EDD).
} 
estudo das respostas $R_{i}^{\natural}$ e das "outras obras" $O_{k}$ necessárias para se utilizar, efetivamente, estas respostas e estas outras obras (teorias, experimentações, etc.). Recriar-se-á então o milieu $M$ sob a seguinte forma: $M=\left\{R_{1}^{\diamond}, R_{2}^{\diamond}, \ldots, R_{n}^{\diamond}, Q_{n+1}, \ldots, Q_{m}, O_{m+1}, \ldots, O_{p}\right\}$. Na sequência, guardaremos em mente o esquema herbartiano expandido assim: $[S(X ; Y ; Q) \rightarrow$ $\left.\left\{R_{1}^{\diamond}, R_{2}^{\diamond}, \ldots, R_{n}^{\diamond}, Q_{n+1}, \ldots, Q_{m}, O_{m+1}, \ldots, O_{p}\right\}\right] \rightarrow R^{\vee}$. As questões $Q_{n+1}, \ldots, Q_{m}$ são ditas geradas pela investigação em curso, que aborda a $Q$, dita geradora da investigação. (Chevallard, 2012-2013, p. 1-2, tradução nossa).

O texto da citação precedente indica que o milieu $M$ é constituído por obras de criações humanas produzidas com intencionalidades e finalidades. Esse mesmo milieu presume uma investigação codisciplinar, gerada por uma questão $Q$, realizável se existirem às questões auxiliares $Q_{n}+1, \ldots, Q_{m}$, intermediando o estudo das respostas $R_{1}^{\diamond}, R_{2}^{\diamond}, \ldots, R_{n}^{\diamond}$ e das "outra obras" $O_{m}+1, \ldots, O_{p}$. Aplica-se a esse milieu $M$ as obras que denotamos, anteriormente, por $\boldsymbol{\vee}_{\mathfrak{D}}, \boldsymbol{\vee}_{\mathfrak{M}}$ e $\boldsymbol{\vee}_{\mathfrak{E} .}$ A ampliação das ideias da investigação codisciplinar está descrita na citação abaixo.

Investigar sobre uma determinada questão é um tipo de tarefas que denotaremos por $H$ (da palavra grega historia que significa "investigar") ao entorno da qual nos esforçaremos para construir uma praxeologia da investigação, $\mathcal{H}$, que se denota por $\mathcal{H}=\left[\begin{array}{llll}H / & \tau_{H} / & \theta_{H} / & \Theta_{H}\end{array}\right]$. O esquema herbartiano $[\mathrm{S}(X ; \quad Y ; \quad Q) \rightarrow$ $\left.\left\{R_{1}^{\diamond}, R_{2}^{\diamond}, \ldots, R_{n}^{\diamond}, Q_{n+1}, \ldots, Q_{m}, O_{m+1}, \ldots, O_{p}\right\}\right] \rightarrow R^{\vee}$ constitui um elemento tecnológico $\left(\in \theta_{H}\right)$ que está no coração desta praxeologia da investigação: esse elemento tecnológico justifica vários gestos técnicos, sobre o qual nos voltaremos, tal como colocar-se a procura da reposta $R^{\diamond}$. (Chevallard, 2012-2013, p. 2, tradução nossa).

O bloco praxeológico $\mathcal{H}$ (Chevallard, 2012-2013) assume o esquema herbartiano expandido como tecnologia $\theta_{H}$ para justificar os gestos técnicos $\tau_{H}$ aplicáveis ao tipo de tarefas $H$. A extensão desse bloco praxeológico alcança o parcours d'étude et de recherche (PER): "Para uma determinada questão $Q$, o trabalho de investigação pode adotar uma grande diversidade de parcours d'étude et de recherche (PER)" (Chevallard, 2012-2013, p. 2, tradução nossa). Temos neste parágrafo uma das abordagens em expansão da TAD que interliga a praxeologia da Investigação Codisciplinar (IC) à praxeologia do PER. Assim,

Em certos tipos de percurso, o investigador ou estudante ignorará, voluntariamente ou não, as respostas $R^{\diamond}$ depositadas na cultura e tentará construir "diretamente" a resposta $R^{\boldsymbol{v}}$ procurada, apoiando-se sobre as obras $O_{m+1}, \ldots, O_{p}$ julgadas por ele apropriadas. Em tal PER, a parte da pesquisa é máxima, enquanto o estudo recai, unicamente, sobre as obras $O_{k}$, que ele está aprendendo usar como ferramenta de pesquisa. 
Em outro tipo de percurso, colocar-se-á, prioritariamente, a busca pelas respostas $R^{\diamond}$ e as questões $Q_{j}$ assim como as outras obras $O_{k}$ mobilizadas terão então por objeto, primeiro ajudar o investigador a estudar as repostas $R_{i}^{\diamond}$ assim reunidas, em vista de "desconstruí-las" e extrair os materiais para construção da resposta $R^{\text {". }}$.

Em todos os casos ou quase, a investigação se desenvolverá de maneira ótima de acordo com um percurso feito de uma parte de estudo e de uma parte de pesquisa, um ao outro combinado nas proporções variáveis segundo a continuidade do PER. (Chevallard, 2012-2013, p. 2, tradução nossa).

Temos na citação duas praxeologias de investigação relacionadas ao PER. A primeira detém-se na busca das respostas autenticadas $\left(R^{\dagger}\right)$ exclusivamente contidas nas obras $O_{k}$, mas estas obras devem estar acessíveis em alguma instituição ou local para a respectiva consulta do investigador - a exemplo da pesquisa documental. A segunda é mais ampla, permite a busca das respostas autenticas em outras obras $O_{k}$, mas essa busca ocorre intermediada pelas questões $Q_{j}$ que auxilia o investigador no estudo dos tipos de tarefas $H_{i}$, ou seja, os tipos de tarefas $H_{1}, H_{2}, H_{3}, H_{4}$ e $H_{5}$. Desses cinco tipos de tarefas $H_{i}$, os três primeiros se relacionam ao estudo das repostas autenticadas $R^{\diamond}$, os dois últimos à construção da resposta procurada $R^{\vee}$.

$H_{1}$. Observar as respostas $R^{\diamond}$ depositadas nas instituições.

$H_{2}$. Analisar - consistentemente, o duplo plano experimental e teórico - estas respostas $R^{\natural}$.

$H_{3}$. Avaliar estas mesmas respostas $R^{\diamond}$.

$H_{4}$. Desenvolver uma resposta própria $R^{\vee}$.

$H_{5}$. Difundir e defender a resposta $R^{\boldsymbol{v}}$ assim produzida (Chevallard, 2012-2013, p. 3, tradução nossa).

De certa forma, uma pesquisa de doutoramento perpassa pelos tipos de tarefas $H_{i}$, principalmente, como filtro para elaboração da resposta ótima $R^{\mathbf{v}}$ para a questão $Q$, que reflita na confirmação da tese. Assim, esboçamos na Figura 1, o escopo teórico simplificado para a Investigação Codisciplinar (IC) e PER. Nessa figura a TAD é a teoria de amplo alcance em diversas áreas da cultura humana e as IC acabam sendo múltiplas também (Chevallard, 2012-2013). Nessa multiplicidade, o dispositivo do PER se adéqua perfeitamente ao bloco praxeológico $\mathcal{H}=\left[H / \tau_{H} / \theta_{H} / \Theta_{H}\right]$ e pela tecnologia $\theta_{H}$ do esquema herbartiano. 


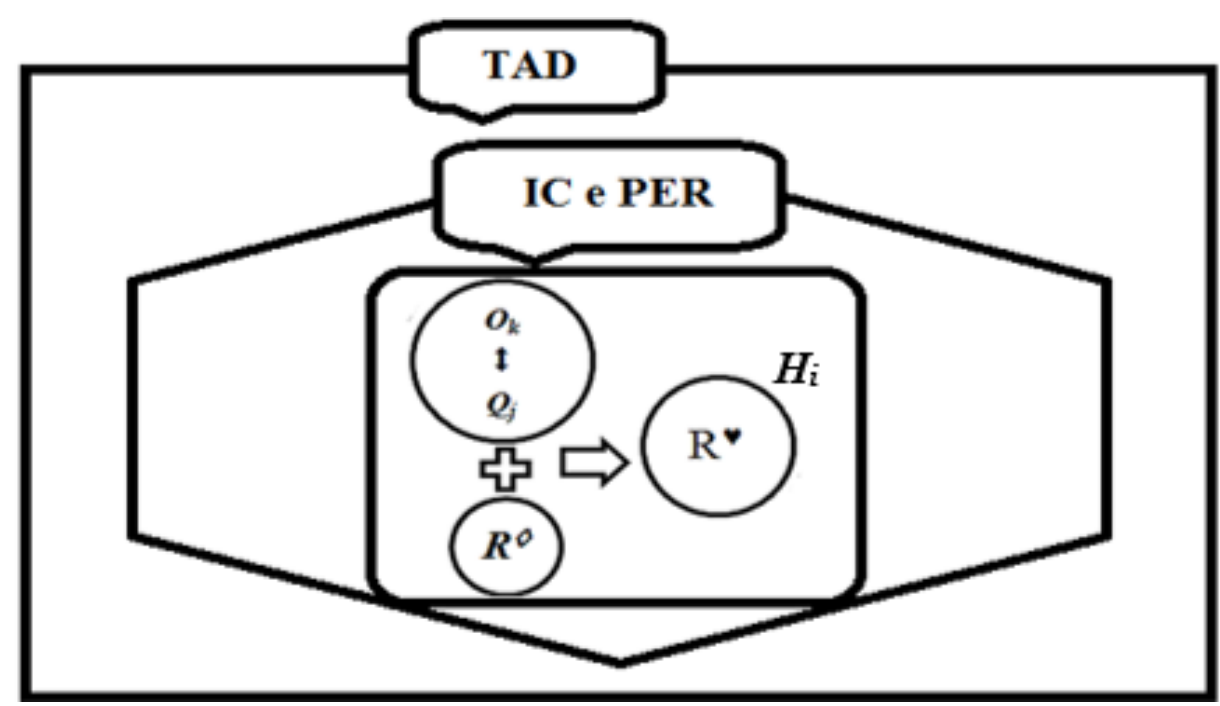

Figura 1 - Esquema teórico simplificado para IC e PER Fonte: Elaborada pelos autores (2016)

Em conexão como esquema teórico da Figura 1, elaboramos quatro esquemas herbartianos (denotados por $\mathrm{W}_{\mathrm{i}}$ ) que nortearam a pesquisa de tese do primeiro autor (obra $\vee$ T). O primeiro é relativo ao início da pesquisa, alinhamos a problemática à revisão da literatura pertinente para continuarmos as outras etapas da pesquisa: Њ1: $\left[S\left(x ; y ; \boldsymbol{\vartheta}_{\tau} \rightleftarrows\right.\right.$ Q) $\rightarrow M] \rightarrow R^{\bullet}$. O segundo com o milieu $M$ definido, temos a mesogênese em fluxo, ou seja, o sistema de recursos que utilizaremos no prosseguimento da pesquisa: Њ2: [S(x; $y$; $\left.\left.\boldsymbol{v}_{\mathrm{T}} \rightleftarrows \mathrm{Q}\right) \rightarrow M\right] \leadsto \mathrm{R}^{\bullet}$. O terceiro índico o avanço da pesquisa intermediado pelo milieu $M \mathrm{e}$ com as possíveis respostas autenticadas, selecionadas das obras $O_{k}$ : Њ3: $[S(x ; y ; \vee \tau \rightleftarrows Q)$ $\rightarrow M] \rightarrow R^{\ominus}$. O último seria a pesquisa finalizada, o coração da resposta $R^{\triangleright}$ é substituído pelo coração $\bullet$, significando que o estudo de obras $O_{k}$ e análise das respostas, $R_{i}^{\diamond}, R_{j}^{\diamond} \mathrm{e}$ $R_{n}^{\diamond}$, levaram a concretização resposta procurada $R^{\vee}: \biguplus_{4}:[S(x ; y ; \bullet \tau \rightleftarrows Q) \rightarrow M] \rightarrow R^{\vee}$. Exibimos no esquema da Figura 2, uma possível praxeologia em vista da elaboração da resposta $R^{\bullet}$. 


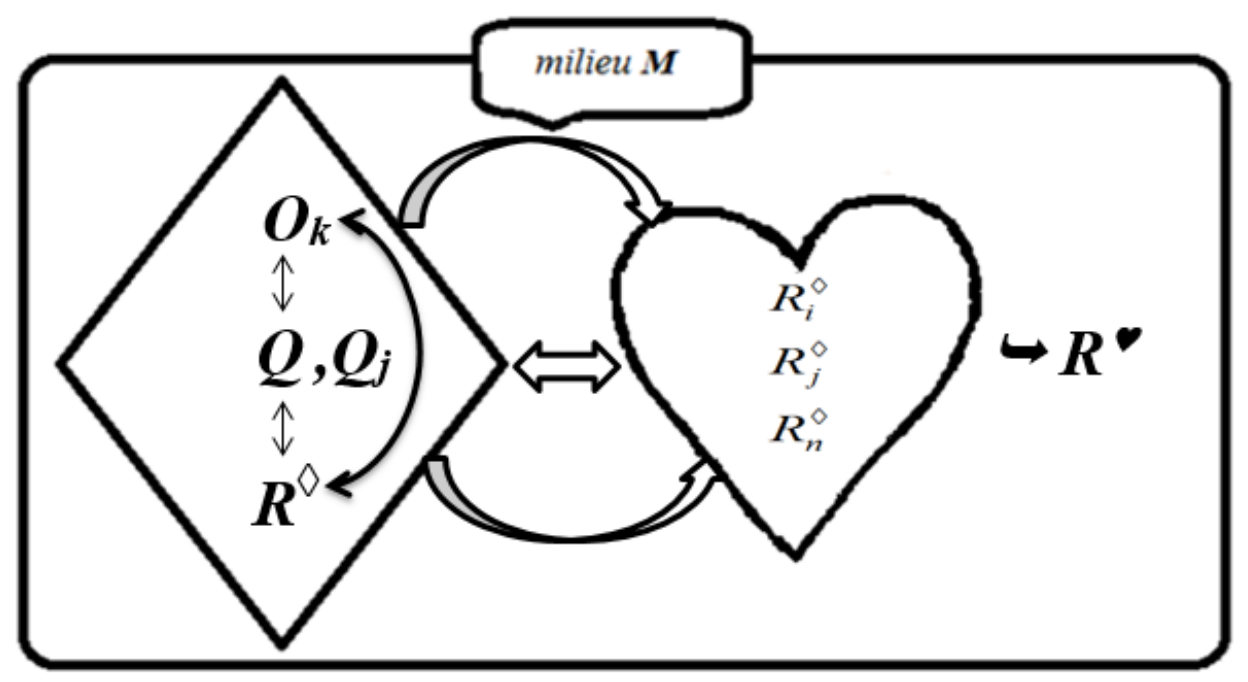

Figura 2 - Possível praxeologia à elaboração da $\mathrm{R}^{\vee}$ Fonte: Elaborada pelos autores (2016)

Os elementos característicos da Figura 2 imputam ao milieu $M$ uma amplitude conforme o significado da palavra na abordagem da TAD. Entenda-se que o esquema esboçado na Figura 2 está em conformidade com o sistema didático $S\left(x ; y ; \boldsymbol{\varphi}_{\tau} \rightleftarrows Q\right)$. $O$ losango ampliado significa a escolha e validação das obras pelo $x$ (doutorando) e $y$ (orientador), em consonância com a questão norteadora $Q$ e outras questões $Q_{j}$ (por exemplo, questões auxiliares $Q_{0}, Q_{1}, Q_{2}$ ). Essas questões impulsionam a identificação das respostas $R^{\diamond}$, contidas nas obras $O_{k}$. Essas respostas $R^{\diamond}$ são autenticadas por categorias $R_{i}^{\diamond}, R_{j}^{\diamond}$ e $R_{n}^{\diamond}$, e, dependem das intencionalidades e finalidades da pesquisa e de quem as propõe.

A escolha das respostas $R_{i}^{\diamond}, R_{j}^{\diamond}$ e $R_{n}^{\diamond}$ estabelece uma dialética com o símbolo do coração em branco (a preencher) e com a resposta $\mathrm{R}^{\circ}$. O preenchimento desse coração imprime uma análise praxeológica preliminar para excluir as respostas autenticadas que divergem da intencionalidade e finalidade da pesquisa conduzida por $x$ (doutorando) e orientada por $y$ (orientador) - concretização dos tipos de tarefas $H_{1}, H_{2}$ e $H_{3}$. Expurgadas essas respostas, restam as que conduzirão $x$ na construção da resposta $R^{\vee}$ para a questão $Q$ - é a vez do tipo de tarefas $H_{4}$ e a substituição do esquema herbartiano Њ3

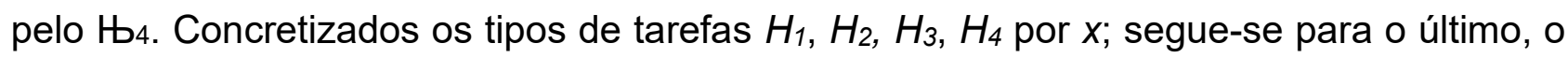
$H_{5}$. Para esse último tipo de tarefas $H_{i}, x$ divulgará e defenderá as respostas $\mathrm{R}^{\vee}$, perante integrantes da comunidade científica nacional $\Xi^{*}$ ou internacional $\Xi^{* *}$ (Chevallard \& Artaud, 2013-2014a). 
Os cinco tipos de tarefas $H_{i}$, que Chevallard (2011d) denominou de "cinco gestos básicos", ganharam novo redimensionamento teórico em Chevallard e Artaud (20132014a), tornando-os elementos praxeológicos dos "Fundamentos e Métodos da Pesquisa em Didática" (Chevallard \& Artaud, 2013-2014a, 2013-2014b) ${ }^{12}$. De acordo com esses autores:

O modelo de pesquisa de referência do pesquisador $\xi$ é definido - de forma evolutiva - pelo conjunto de questões às quais ele se mostra capaz de dar respostas. Uma parte desse modelo está evoluindo lentamente: é a teoria do didático sobre a qual o pesquisador se apoia - é para nós, aqui, a TAD. (Chevallard \& Artaud, 2013-2014b, p. 21, tradução nossa).

Assim, nossa compreensão estabelece que o fluxo praxeológico das cinco $H_{i}$ devem permear as praxeologias das pesquisas no âmbito da Didática da Matemática, principalmente, no contexto da TAD.

\section{CONSIDERAÇÕES FINAIS}

A dimensão científica alcançada pela Teoria Antropológica do Didático (TAD) ultrapassou o contexto das pesquisas em Didática da Matemática ou Educação Matemática. Porém, a complexidade teórica da TAD demanda produções textuais para torná-la mais compreensível e, principalmente, revelar como essa teoria é aplicada em pesquisas científicas. Nesse sentido, neste artigo, expusemos algumas abordagens da TAD, entre as quais tratamos do significado de obras, sistemas didáticos, esquema herbartiano, milieu e várias simbologias inerentes a própria teoria.

Em termos de relevância, o estudo de obras é algo que pontuamos como essencial para o desenvolvimento de pesquisas científicas quando se escolhe a TAD para fundamentar as etapas de uma pesquisa de doutoramento. Em relação a isso, temos a compreensão que as etapas praxeológicas da pesquisa, quando se apoiam na TAD, ganham fluxo por intermédio de esquemas herbartiano.

Outro aspecto que permeia a TAD é a Investigação Codisciplinar (IC) em conexão ao dispositivo metodológico do parcours d'étude et de recherche (PER) [percurso de estudo e pesquisa (PEP)]. Na Figura 1, mostramos, resumidamente, a potencialidade dessa conexão entre IC e PER, ou seja, o bloco praxeológico $\mathcal{H}=\left[H / \tau_{H} / \theta_{H} / \Theta_{H}\right]$ possui a tecnologia $\theta_{H}$ pertinente ao esquema herbartiano, que auxilia no estudo das obras $O_{k}$ e

12 Fondements et méthodes de la recherche en didactique - Leçon 3 ; Leçon 4. 
escolhas das respostas $R^{\diamond}$ para as questões $Q_{j}$. Essa praxeologia de estudo e escolha impulsiona o pesquisador na aplicação das técnicas $\tau_{H}$ para os tipos de tarefas $H_{i}$.

A mesogênese que consta na Figura 2 resume os elementos teóricos do milieu $M$ mobilizados para determinada pesquisa científica que se apoia na TAD. O efeito dessa mesogênese possui uma proposição teórica-simbólica do coração "vazio" (em branco), que, simbolicamente, deve ser preenchido a partir da análise e escolha das respostas $R_{i}^{\diamond}, R_{j}^{\diamond}$ e $R_{n}^{\diamond}$, tornando possível a elaboração da resposta procurada $\mathrm{R} \bullet$.

Assim, vemos que o propósito maior deste artigo está concluído, ou seja, expor nossas proposições teórico-simbólicas mediadas pela Teoria Antropológica do Didático, as quais entendemos ter relevância para futuras pesquisas em Educação Matemática.

\section{REFERÊNCIAS}

Briant, Nathalie (2013). Étude didactique de la reprise de l'algèbre par l'introduction de l'algorithmique au niveau de la classe de seconde du lycée français. Thèse pour obtenir le grade de Docteur - Délivré par UNIVERSITE MONTPELLIER 2. Disponível em: https://tel.archives-ouvertes.fr/tel-00920506v1. Acesso em: 10 maio 2014.

Carvalho, Cristiane C.; \& Pereira, José C. S. (2009). Aprendizagem significativa - das operações aritméticas às operações algébricas: o tratamento das operações algébricas a partir das operações aritméticas como conhecimento prévio. Monografia (Especialização em Educação Matemática). Núcleo de Pesquisa e Desenvolvimento da Educação Matemática e Científica da Universidade Federal do Pará. Belém.

Catalán, Pilar Bolea (2003). El proceso de algebrización de organizaciones matemáticas escolares. Zaragoza: Prensas Universitarias de Zaragoza: Departamento de Matemática Aplicada, Universidad de Zaragoza (Monografias del Seminário Matemático "Garcia de Galdeano", 29). Tesis - Universidad de Zaragoza.

Chevallard, Yves (2007). Didactique de L'Enquête Codisciplinaire. Journal du Seminaire TAD/IDD $\quad-\quad 1$ 1; 15 -29 http://yves.chevallard.free.fr/spip/spip/IMG/pdf/journal-du-seminaire-tad-idd-1.pdf Acesso em: 27 mar. 2016.

Chevallard, Yves (1998). Analyse des pratiques enseignantes et didactique des mathématiques: l'approche anthropologique. Disponível em: http://yves.chevallard.free.fr/spip/spip//MG/pdf/Analyse des pratiques enseignantes.p df . Acesso em: 15 jun. 2015.

Chevallard, Yves (2002). Approche anthropologique du rapport au savoir et didactique des mathematiques.

em: 
http://yves.chevallard.free.fr/spip/spip/IMG/pdf/Approche anthropologique rapport au savoir.pdf. Acesso em: 02 jun. 2012.

Chevallard, Yves (2013). Éléments de didactique du développement durable - Leçon 1: Enquête codisciplinaire \& EDD. 2012-2013. Disponível em: http://yves.chevallard.free.fr/spip/spip/IMG/pdf/Didactique du DD 2012-2013 1.pdf.

Acesso em: 07 jul. 2016.

Chevallard, Yves (1988). Esquisse d'une théorie formelle du didactique. Disponivel em: http://yves.chevallard.free.fr/spip/spip/lMG/pdf/Esquisse d une theorie formelle du didactique.pdf. Acesso em: 15 jun. 2015.

Chevallard, Yves (2011a). Introdução à teoria antropológica do didático [bilingue]. Disponível em: http://yves.chevallard.free.fr/spip/spip/article.php3?id article=210. Acesso em: 24 jun. 2015.

Chevallard, Yves (2009c). La notion de PER: problèmes et avancées. Disponível em: http://yves.chevallard.free.fr/spip/spip/article.php3?id article=161. Acesso em: 24 jun. 2013.

Chevallard, Yves (2009a). La TAD face au professeur de mathématiques. Disponível em: http://yves.chevallard.free.fr/spip/spip/article.php3?id article=162. Acesso em: 24 abr. 2011.

Chevallard, Yves (2011d). Le Mathématique et le Didactique. In: Journal du Seminaire TAD/IDD- 2010/2011-4; 1-15. Disponivel em: http://yves.chevallard.free.fr/spip/spip/IMG/pdf/journal-tad-idd-2010-2011-4.pdf.

Acesso em: 27 mar. 2016.

Chevallard, Yves (1994). Les processus de transposition didactique et leur théorisation. Disponivel em: http://yves.chevallard.free.fr/spip/spip/article.php3?id article=114. Acesso em: 27 mar. 2016.

Chevallard, Yves (2009b). Notes pour un Exposé à Venir. Journal du Seminaire TAD/IDD - 6; 1-20. Disponível em: http://yves.chevallard.free.fr/spip/spip/IMG/pdf/journal-tadidd-2008-2009-6.pdf. Acesso em: 27 mar. 2016.

Chevallard, Yves (2011c). Profession, chercheur en didactique. Journal du Seminaire TAD/IDD-2011/2012-2; 1 1-9. Disponível em: http://yves.chevallard.free.fr/spip/spip/IMG/pdf/journal-tad-idd-2011-2012-2.pdf. Acesso em: 27 mar. 2016.

Chevallard, Yves (2009d). Remarques sur la notion d'infrastructure didactique et sur le rôle des PER. Disponível em: http://yves.chevallard.free.fr/spip/spip/article.php3?id article=155. Acesso em: 24 jun. 2013.

Chevallard, Yves (2011b). Sigisbées et sycophantes. Journal du Seminaire TAD/IDD2010/2011-3 $1-14$.

Disponível em: 
http://yves.chevallard.free.fr/spip/spip/IMG/pdf/journal-tad-idd-2010-2011-3.pdf.

Acesso em: 27 mar. 2016.

Chevallard, Yves (2008). Sur la TAD. Journal du Seminaire TAD/IDD - 8; 7-25. Disponível em: http://yves.chevallard.free.fr/spip/spip/IMG/pdf/journal-du-seminaire-tad-idd-8.pdf. Acesso em: 27 mar. 2016.

Chevallard, Yves; \& Artaud, Michèle (2014a). Fondements et méthodes de la recherche en didactique - Leçon 3: Praxéologies de recherche I. 2013-2014. Disponível em: http://yves.chevallard.free.fr/spip/spip/IMG/pdf/DDM - UE 35 - YC - Lecons 3.pdf. Acesso em: 15 jun. 2015.

Chevallard, Yves; \& Artaud, Michèle (2014b). Fondements et méthodes de la recherche en didactique - Leçon 4: Praxéologies de recherche II. 2013-2014. Disponível em: http://yves.chevallard.free.fr/spip/spip/IMG/pdf/DDM - UE 35 - YC - Lecon 4.pdf. Acesso em: 15 jun. 2015.

Chevallard, Yves; \& Bosch, Marianna (2012). L'algebre entre effacement et reaffirmation aspects critiques de l'offre scolaire d'algebre. In: Coulange, Lalina et al. Enseignement de l'Algèbre Élémentaire: bilan et perspectives. Hors série - Revue Recherches en didactique des mathématiques. France: Éditions la Pensée Sauvage, 19-39.

Delgado, Tomás Ángel Sierra (2006). Lo Matemático en el Diseño y Analisis de Organizaciones Didácticas: los sistemas de numeración y la medida de magnitudes. Memoria para optar al Grado de Doctor. Universidad Complutense de Madrid, Facultad de Educación, Departamento de Didáctica y Organización Escolar. Madrid. Dispomível em: https://eprints.ucm.es/7373/. Acesso em: 02 jun. 2012.

Euler, Léonard (1795). Élémens d'algèbre. Lyon. Disponível em: http://www.erara.ch/doi/10.3931/e-rara-8611. Acesso em: 14 ago. 2014.

Farras, B. B.; Bosch, M.; \& Gascón, J. (2013). Las tres dimensiones del problema didáctico de la modelización matemática. Revista Educação Matemática e Pesquisa, v. 15,1 n. $1, \quad 1-28 . \quad$ Disponível em: http://revistas.pucsp.br/index.php/emp/article/view/12757. Acesso em: 07 set. 2014.

Gascón, Josep (2011). Las tres dimensiones fundamentales de um problema didáctico: el caso del álgebra elemental. Revista Latinoamericana de Investigación em Matemática Educativa, v. 14, n. 2, 203-231. Disponível em: https://www.redalyc.org/pdf/335/33519238004.pdf. Acesso em: 07 set. 2014.

Girard, Albert $(1629,1884)$. Invention nouvelle en l'algèbre. Chez Cuillaume lansson Bleuw. Amsterdam, 1629. Reimpression par Dr. Bierens de Hann. Leiden. Disponível em: http://dx.doi.org/10.3931/e-rara-4803. Acesso em: 30 jul. 2014.

Hilgenheger, Norbert (2010). Johann Herbart. Trad. José Eustáquio Romão. Recife: Fundação Joaquim Nabuco, Editora Massangana. Disponível em: http://www.dominiopublico.gov.br/download/texto/me4672.pdf. Acesso em: 27 mar. 2016. 
Maclaurin, Colin (1753). Traité d'algèbre et de la manière de l'appliquer. Paris. Disponível em: http://gallica.bnf.fr/ark:/12148/bpt6k1095477. Acesso em: 31 jul. 2014.

Matheron, Yves (2000). Une Etude Didactique de la Memoire nans l'Enseignement des Mathematiques au College Et au Lycee. Quelques Exemples. These pour obtenir le grade de DOCTEUR DE L'UNIVERSITE AIX-MARSEILLE I. Disponível em : https://tel.archives-ouvertes.fr/tel-00586312. Acesso em: 30 jul. 2014.

Matos, Fernando Cardoso de (2017). Praxeologias e modelos praxeológicos institucionais: o caso da álgebra linear. Tese (Doutorado) - Universidade Federal do Pará, Instituto de Educação Matemática e Científica, Programa de Pós-Graduação em Docência em Educação em Ciências e Matemáticas, Belém.

Pereira, J. C. S.; \& Nunes, J. M. V. (2017). Ensino de operações polinomiais intermediado pela aritmética no sistema de numeração posicional decimal. Educação Matemática Pesquisa, São Paulo, v.19, n.1, 251-271. Disponível em: https://revistas.pucsp.br/emp/article/view/27152. Acesso em: 05 mar. 2019.

Pereira, J. C. S. (2017). Alterações e recombinações praxeológicas reveladas por professores de matemática do ensino básico em formação continuada: a partir de um modelo epistemológico alternativo para o ensino da álgebra escolar. Tese de Doutorado. Universidade Federal do Pará, Instituto de Educação Matemática e Científica, Programa de Pós-Graduação em Educação em Ciências e Matemáticas. Belém.

Pereira, J. C. S. (2012). Análise Praxeológica de Conexões entre Aritmética e Álgebra no Contexto do Desenvolvimento Profissional do Professor de Matemática. Dissertação (Mestrado). Universidade Federal do Pará, Instituto de Educação Matemática e Científica, Programa de Pós-Graduação em Educação em Ciências e Matemáticas. Belém.

Viète, François (1630). Introduction en l'art analytic, ou nouvelle algèbre. Paris. Disponível em: http://dx.doi.org/10.3931/e-rara-4788. Acesso em: 30 jul. 2014.

\section{NOTAS}

\section{TÍTULO DA OBRA}

Proposições teórico-simbólicas mediadas pela teoria antropológica do didático

José Carlos de Souza Pereira

Doutor em Educação em Ciências e Matemáticas pelo Programa de Pós-Graduação em Educação em Ciências e Matemáticas (PPGECM) do Instituto de Educação Matemática e Científica (IEMCI) da Universidade Federal do Pará (UFPA)

Secretaria de Educação do Estado do Pará - SEDUC/PA, Belém - PA, Brasil

jsouzaper@gmail.com

Dhttps://orcid.org/0000-0003-4797-0023

José Messildo Viana Nunes

Doutor em Educação Matemática pela Pontifícia Universidade Católica de São Paulo (PUC/SP). Professor do Programa de PósGraduação em Educação em Ciências e Matemáticas (PPGECM) do Instituto de Educação Matemática e Científica (IEMCI) da Universidade Federal do Pará (UFPA), Belém - PA, Brasil

messildo@ufpa.br

Dhttps://orcid.org/0000-0001-9492-4914

Endereço de correspondência do principal autor

Travessa Nove de Janeiro, 3326, CEP: 66065-155, Bairro: Condor, Belém, PA, Brasil 


\section{AGRADECIMENTOS}

A Coordenação do Programa de Pós-Graduação em Educação em Ciências e Matemáticas (PPGECM) do Instituto de Educação Matemática e Científica (IEMCI), que oportunizou a realização da pesquisa de doutoramento do primeiro autor.

Ao Grupo de Estudos e Pesquisas da Didática da Matemática - GEDIM/PPGECM/UFPA, que realiza estudos e pesquisas em Didática da Matemática, possibilitando que graduandos, pós-graduandos e pesquisadores atuam em cooperação para o avanço das pesquisas em Educação Matemática na Região Amazônica e no Brasil.

\section{CONTRIBUIÇÃO DE AUTORIA}

Concepção e elaboração do manuscrito: J. C. S. PEREIRA.

Coleta de dados: J. C. S. PEREIRA.

Análise de dados: J. C. S. PEREIRA, J. M. V. NUNES.

Discussão dos resultados: J. C. S. PEREIRA, J. M. V. NUNES.

Revisão e aprovação: J. C. S. PEREIRA, J. M. V. NUNES.

\section{CONJUNTO DE DADOS DE PESQUISA}

Todo o conjunto de dados que dá suporte aos resultados deste estudo foi publicado no próprio artigo.

\section{FINANCIAMENTO}

Não se aplica.

\section{CONSENTIMENTO DE USO DE IMAGEM}

Não se aplica.

\section{APROVAÇÃO DE COMITÊ DE ÉTICA EM PESQUISA \\ Não se aplica.}

\section{CONFLITO DE INTERESSES}

Não se aplica.

\section{LICENÇA DE USO}

Os autores cedem à Revemat os direitos exclusivos de primeira publicação, com o trabalho simultaneamente licenciado sob a Licença Creative Commons Attribution (CC BY) 4.0 International. Estra licença permite que terceiros remixem, adaptem e criem a partir do trabalho publicado, atribuindo o devido crédito de autoria e publicação inicial neste periódico. Os autores têm autorização para assumir contratos adicionais separadamente, para distribuição não exclusiva da versão do trabalho publicada neste periódico (ex.: publicar em repositório institucional, em site pessoal, publicar uma tradução, ou como capítulo de livro), com reconhecimento de autoria e publicação inicial neste periódico.

\section{PUBLISHER}

Universidade Federal de Santa Catarina. Grupo de Pesquisa em Epistemologia e Ensino de Matemática (GPEEM). Publicação no Portal de Periódicos UFSC. As ideias expressadas neste artigo são de responsabilidade de seus autores, não representando, necessariamente, a opinião dos editores ou da universidade.

\section{EDITOR}

Méricles Thadeu Moretti e Rosilene Beatriz Machado.

\section{HISTÓRICO}

Recebido em: 25-03-2020 - Aprovado em: 30-07-2020 